\title{
Nutzen von Bosentan bei Patienten mit PH und IIP
}

\author{
Ob Bosentan auch Patienten hilft, bei denen eine pulmonale \\ Hypertonie (PH) mit gleichzeitiger interstitieller Lungen- \\ erkrankung besteht, konnte aufgrund der unzureichenden \\ Datenlage bislang nicht beantwortet werden. Daher haben \\ T. J. Corte et al. erstmals eine Studie zu dieser Fragestellung \\ durchgeführt.
} Am J Respir Crit Care Med 2014; 190: 208-217

Die in die Studie aufgenommenen Patienten hatten eine mit Rechtsherzkatheter bestätigte $\mathrm{PH}$ und litten entweder an idiopathischer pulmonaler Fibrose (IPF) oder nichtspezifischer interstitieller Pneumonie (NSIP). Mindestens 3 Monate vor Studienaufnahme durften die Teilnehmer keine PH-spezifischen Medikamente mehr einnehmen. Bosentan und Placebo wurden über 16 Wochen 2-mal täglich oral eingenommen. Zu Beginn betrug die Verumdosis 62,5 mg (2-mal täglich), die nach einem Monat auf $125 \mathrm{mg}$ erhöht werden konnte. Insgesamt wurden 60 Patienten, davon 42 Männer, mit einem Durchschnittsalter von 66,6 Jahren rekrutiert. Bei 46 Teilnehmer bestand eine IPF-Diagnose und 14 hatten NSIP. Das primäre Studienziel war eine Abnahme des pulmonalen Gefäßwiderstandsindex (Pulmonary Vascular Resistance Index, PVRI) gegenüber dem Ausgangswert um $\geq 20 \%$ über 16 Wochen. Die sekundären Endpunkte umfassten funktionelle Kapazität und Lebensqualität, Hämodynamik, Sauerstoffsättigung und -bedarf sowie Krankheitsprogression.

Zu Studienbeginn betrug der mittlere pulmonal-arterielleDruck(mPAP)36,0 mmHg, der PVRI 13 Wood-Einheiten $/ \mathrm{m}^{2}$ und der Herzindex (HI) $2,21 \mathrm{l} / \mathrm{min} / \mathrm{m}^{2}$. Der $\mathrm{Zu}-$ stand von 26 Patienten entsprach der funktionellen WHO-Klasse 3 und von 30 Patienten in der WHO-Klasse 4. Die Durchschnittswerte für die FVC von 54,2\% und für den CPI (zusammengesetzter physiologischer Index) von 67,8 wiesen auf eine fortgeschrittene idiopathische interstitielle Pneumonie (IIP) hin. Aufgrund von Studienabbrüchen und Tod standen für die Auswertung Daten von 39 Patienten (Bosentan $n=25$, Placebo $n=4$ ) zur Verfügung.

\section{Therapie ohne deutlichen Effekt $\nabla$}

Den primären Endpunkt erreichten in der Bosentan-Gruppe 28\% der Patienten und in der Placebogruppe $28,6 \%$, was in einem p-Wert von 0,97 resultierte. Auch in den sekundären Endpunkten ergaben sich zwischen Verum und Placebo keine statistisch signifikanten Unterschiede. So reduzierte sich die 6-Minuten-Gehstreckte um 25,9 Meter (Bosentan) bzw. 53,1 Meter (Placebo, $p=0,42$ ). Während unter Verum der PVRI um 1,14 Wood-Einheiten $/ \mathrm{m}^{2}$ abnahm, stieg er unter Placebo um 0,83 Wood-Einheiten $/ \mathrm{m}^{2}(\mathrm{p}=0,19)$. Der mPAP sank in der Bosentan-Gruppe um $1,31 \mathrm{mmHg}$ und nahm unter Placebo um $0,21 \mathrm{mmHg} z u(p=0,43)$. Die Unterschiede bei der Sauerstoffsättigung, dem Sauerstoffbedarf sowie dem Krankheitsprogress blieben mit p-Werten von 0,79, 0,08 sowie 0,47 unter der statistischen Signifikanz. Das galt auch für die Subgruppenanalysen.

\section{Fazit}

Der Endothelin-Rezeptorantagonist Bosentan kann Kollagenablagerungen in der Lunge reduzieren und bei pulmonalarterieller Hypertonie die Hämodynamik und funktionelle Kapazität verbessern. Patienten mit IIP und PH profitieren nach den Ergebnissen dieser Studie nicht von einer Bosentan-Therapie. $\mathrm{Zu}$ keinem Studienendpunkt wurde ein statistisch signifikanter Unterschied gegenüber Placebo erreicht. Die Autoren können Bosentan für diese Patienten nicht empfehlen.

Matthias Manych, Berlin
Forschung

\section{Genvariante schützt vor Lungenhochdruck}

Ein internationales Forscherteam konnte unter Beteiligung von Wissenschaftlern der Technischen Universität Braunschweig wichtige Ergebnisse für die Behandlung des Lungenhochdrucks erzielen. Bei Untersuchungen im zentralasiatischen PamirGebirge fanden sie heraus, dass Teile der dort ansässigen kirgisischen Volksgruppe vor der lebensbedrohlichen Erkrankung durch einen genetischen Vorteil geschützt sind. Als Folge von Lungenerkrankungen kann der erhöhte Blutdruck im Lungenkreislauf zu Herzversagen führen, erklärt Prof. Sönke Behrends. „Erhalten Teile der Lunge zu wenig Sauerstoff, reagiert der Körper dort mit einer Verengung der Blutgefäße, um zu verhindern, dass das sauerstoffarme Blut in den Körperkreislauf gelangt. Solange andere Abschnitte der Lunge ausreichend mit Sauerstoff versorgt sind, ist das ein sinnvoller Mechanismus. Wenn aber die ganze Lunge zu wenig Sauerstoff erhält, wird es problematisch. Dann verengen sich alle Blutgefäße in der Lunge und das Herz kann schweren Schaden nehmen“, erläutert der Pharmakologe. Laut der Ende 2014 in der Fachzeitschrift Circulation publizierten Studie weist ein Teil der untersuchten Kirgisen eine natürliche Genvariante auf. Dadurch seien sie für einen körpereigenen Botenstoff besonders empfindlich, der für die Erweiterung der Blutgefäße in der Lunge zuständig ist, erläutert Behrends. „Nun sehen wir, dass Unterschiede im Erbgut die Effizienz dieses Botenstoffes erhöhen können. Der entschlüsselte Genabschnitt beeinflusst den Angriffspunkt für ein neuartiges Medikament gegen den Lungenhochdruck, das seit 2014 auch in Deutschland erhältlich ist“, so Behrends. Die Wissenschaftler versprechen sich von den Ergebnissen daher auch bessere Möglichkeiten zur Behandlung des Lungenhochdrucks. Denn bisher gibt es nur wenige Therapiemöglichkeiten für die lebensbedrohliche Erkrankung. „Die Unterschiede im Erbgut ergeben einen neuen Ansatzpunkt für eine auf den einzelnen Patienten zugeschnittenen Therapie. Statt Medikamente einfach auszuprobieren, kann eine genetische Analyse des Angriffspunktes oft richtige Voraussagen über die Wirksamkeit machen.“

Nach einer Mitteilung der TU Braunschweig 